\title{
Biochemical Support for the "Threshold" Theory of Creativity: A Magnetic Resonance Spectroscopy Study
}

\author{
Rex E. Jung, ${ }^{1,2,3,4}$ Charles Gasparovic, ${ }^{1,3}$ Robert S. Chavez, ${ }^{1}$ Ranee A. Flores, ${ }^{1}$ Shirley M. Smith,,${ }^{1,3}$ Arvind Caprihan, ${ }^{1}$ and \\ Ronald A. Yeo ${ }^{3}$ \\ ${ }^{1}$ The Mind Research Network, and Departments of ${ }^{2}$ Neurology, ${ }^{3}$ Psychology, and ${ }^{4}$ Neurosurgery University of New Mexico, Albuquerque, New Mexico
} 87131

\begin{abstract}
A broadly accepted definition of creativity refers to the production of something both novel and useful within a given social context. Studies of patients with neurological and psychiatric disorders and neuroimaging studies of healthy controls have each drawn attention to frontal and temporal lobe contributions to creativity. Based on previous magnetic resonance (MR) spectroscopy studies demonstrating relationships between cognitive ability and concentrations of $\mathrm{N}$-acetyl-aspartate (NAA), a common neurometabolite, we hypothesized that NAA assessed in gray and white matter (from a supraventricular slab) would relate to laboratory measures of creativity. MR imaging and divergent thinking measures were obtained in a cohort of 56 healthy controls. Independent judges ranked the creative products of each participant, from which a "Composite Creativity Index" (CCI) was created. Different patterns of correlations between NAA and CCI were found in higher verbal ability versus lower verbal ability participants, providing neurobiological support for a critical "threshold" regarding the relationship between intelligence and creativity. To our knowledge, this is the first report assessing the relationship between brain chemistry and creative cognition, as measured with divergent thinking, in a cohort comprised exclusively of normal, healthy participants.
\end{abstract}

\section{Introduction}

Despite agreement on its role in human achievement, the neurobiological underpinnings of creativity are poorly understood. However, there exists myriad cognitive skills necessary to produce something both "novel and useful," and these skills are manifested within different domains (e.g., visual art vs scientific discovery) (Dietrich, 2004). Although complex, the neuroscientific inquiry of creativity is amenable to the tools of the cognitive neurosciences, meaningfully linking behavior to activity within and between brain networks. The main challenge is not to fall prey to the facile simplifications that invariably arise when discussing creativity (Dietrich, 2007).

Creative achievers tend to be prolific, a fact postulated to conform to a constrained, stochastic behavior "accurately modeled as a quasi-random combinatorial process" (Simonton, 2003). Divergent thinking (DT) tasks, requiring subjects to produce multiple (i.e., prolific) solutions to target problems, have long been used as "proxy" measures of creativity in laboratory settings. Although DT tasks have been relied on heavily within the field of creativity research, they are not synonymous with creativity, and concerted attempts are being undertaken to move beyond reliance on these tasks to assess creative capacity in laboratory settings (Dietrich, 2007). Another issue with neurobiological impli-

\footnotetext{
Received Feb. 4, 2009; accepted March 18, 2009.

This research was funded by a grant from the John Templeton Foundation entitled "The Neuroscience of Creativity."

Correspondence should be addressed to Dr. Rex E. Jung, Pete and Nancy Domenici Hall, MSC11 6040, 1101 Yale NE, Albuquerque, NM 87131. E-mail: rjung@mrn.org.

DOI:10.1523/JNEUROSCI.0588-09.2009

Copyright $\odot 2009$ Society for Neuroscience $\quad$ 0270-6474/09/295319-07\$15.00/0
}

cations (Gray and Thompson, 2004; Jung and Haier, 2007) is whether creativity is best construed as an aspect of intelligence or as an unique ability separate from intelligence. Indeed, many researchers agree that creativity and intelligence are correlated up to a certain threshold [around an intelligence quotient (IQ) of 120] after which they tend to vary independently (Sternberg, 2005).

The majority of psychometric studies in creativity emerged in the latter half of the 20th century, but little progress has been made in identifying brain correlates until quite recently (Dietrich, 2004). EEG studies show that creative individuals exhibit lower levels of mental activity when engaged in the solution of creative problems (i.e., transient hypofrontality) (Jausovec, 2000; Dietrich, 2003). Both increased coherence during "rest" with eyes open (Jausovec and Jausovec, 2000) and stronger centroparietal $\alpha$ synchronization (Fink and Neubauer, 2006) have been linked to creative output and originality. Functional neuroimaging studies have shown both positive and negative activations associated with performance of creative tasks across a wide range of brain regions (Carlsson et al., 2000; Bechtereva et al., 2004; Howard-Jones et al., 2005; Asari et al., 2008), likely confounded by specific task demands and cohort characteristics.

Proton magnetic resonance spectroscopy $\left({ }^{1} \mathrm{H}-\mathrm{MRS}\right)$ is an imaging technique that allows for the assay of neurochemistry in vivo. Within the proton spectrum, $N$-acetylaspartate (NAA) is the most prominent peak, and has been frequently used as a marker of neuronal integrity (Moffett et al., 2007). Greater NAA concentration has been found to predict higher cognitive function in both normal and patient populations (Ross and Sachdev, 2004). 
Here, we used ${ }^{1} \mathrm{H}$-MRS imaging $\left({ }^{1} \mathrm{H}\right.$ MRSI) to evaluate whether NAA concentration is related to creative ability in a normal cohort, and whether such biochemical relationships conformed to the threshold hypothesis.

\section{Materials and Methods}

Sample. The sample consisted of 56 participants, recruited from postings at the University of New Mexico, ranging in age from 18 to 39 (30 males, 26 females). All participants signed a consent form approved by the institutional review board of the University of New Mexico before participation in the experimental protocol. Participants were screened by a clinical neuropsychologist (R.E.J.) and determined to be free of neurological and psychological disorders that would impact experimental hypotheses. Participants were also screened for conditions that would prohibit undergoing a MR imaging scan (e.g., metal implant, orthodontic braces, severe claustrophobia).

Behavioral measures. First, three DT tasks were administered: free condition, four line condition of the design fluency test (DFT), and uses of objects test (UOT) (Lezak et al., 2004). In the free condition of the DFT, subjects are instructed to draw as many unique designs as they could in a period of $5 \mathrm{~min}$; during the four line condition they are constrained in drawing designs comprised of certain types of lines (e.g., straight, dots, curved) during 4 min; during the UOT, subjects are given 1 min to produce as many novel and creative uses as they can think of for common objects (e.g., paper clip). All of these tasks tap DT, the aspect of creativity best assessed in laboratory settings.

Three independent judges ranked the DT products of each participant using the consensual assessment technique (Amabile, 1982) from which a "Composite Creativity Index" (CCI) was derived. The college-aged raters had excellent inter-rater reliabilities across the three measures of DT $($ ICC $=0.81-0.94)$. Rankings for each subject were averaged across the three measures to form the CCI, then $z$-transformed (mean $=0$, $\mathrm{SD}=1$ ), and then converted to a standard score (mean of 100, SD of 15) to create the CCI. We also measured overall fluency of output across the three DT measures by simply tallying number of responses for the UOT, and the free and four line conditions of the DFT.

Second, general verbal fluency, independent of performance on DT measures, was assessed with the Controlled Oral Word Association Test (COWAT), in which the subject is instructed to generate as many words beginning with a given letter of the alphabet in three 1 min trials (Lezak et al., 2004).

Third, participants were administered the Wechsler Abbreviated Scale of Intelligence (WASI) to assess intellectual functioning. The WASI consists of four subtests: vocabulary and similarities, which produce a verbal IQ (VIQ), block design, and matrix reasoning, which produce a performance IQ (PIQ). Based on all four subtests one can derive the full scale IQ (FSIQ).

Fourth, personality was assessed with the NEO Five-Factor Inventory (NEO-FFI). The NEO-FFI is a self-administered measure of normal personality functioning, which produces summary scores across five domains: neuroticism, extraversion, openness, agreeableness, and conscientiousness.

Spectroscopic acquisition. MR examinations were performed on a $1.5 \mathrm{~T}$ Siemens Sonata scanner using an eight-channel phased array head coil. MR spectroscopy was obtained using two-dimensional (2D) Chemical Shift Imaging (CSI) prescribed from the supraventricular slice of a T1weighted sagittal image [field of vision $=200 \times 200 \mathrm{~mm}$, repetition time $/$ echo time $=1500 / 135 \mathrm{~ms}$, voxel size $=8.3 \times 8.3 \times 15.0 \mathrm{~mm}, 9: 32$. The PRESS CSI volume of interest (VOI), was prescribed immediately above the lateral ventricles and parallel to the anterior commissureposterior commissure line (Fig. 1). The size of the VOI was $\sim 75 \pm 5 \mathrm{~mm}$ left to right and $90 \pm 5$ anterior to posterior. Both water suppressed and water unsuppressed spectra were acquired.

Analysis. Spectra were zero-filled to $32 \times 32$ points in k-space, applying a Hamming filter with a 50\% window width, and 2D spatial Fourier transformation (FT). LCmodel was used for postprocessing of spectra. Eight regions of interest (Fig. 2) were identified by two independent raters (R.C., R.B.). Tissue segmentation allowed calculation of the exact proportion of gray and white matter in each of the four regions and correction of metabolic values based on percentage of tissue values (gray, white, and CSF). The predominantly gray matter regions were within the dorsal cingulate gyrus (Brodmann area 24 and 32) and the posterior cingulate (Brodmann area 31).

Statistical analysis. Pearson correlations and stepwise linear regression analyses were conducted in SPSS (Version 13.0 for Windows). As our hypotheses address lateralized, anterior/posterior, and gray/white differences impacting biochemical correlates of behavioral measures, we chose to combine spectroscopic voxels into eight major regions. Accordingly, Bonferroni correction was made for multiple comparisons with significance level set at $0.05 / 8=0.00625$ for the regression analysis. Regressions were conducted with composite spectroscopic measures of NAA [left anterior white matter (LAWM), left anterior gray matter (LAGM), right anterior white matter (RAWM), right anterior gray matter (RAGM), left posterior white matter (LPWM), left posterior gray matter (LPGM), right posterior gray matter (RPGM), right posterior white matter $(\mathrm{RPWM})]$ serving as independent variables and either CCI or fluency (total number of responses generated; COWAT) serving as the dependent measures. Post hoc analyses were performed to establish whether CCI-Fluency-NAA relationships were mediated by intelligence, with a total within family significance threshold for these regression equations 


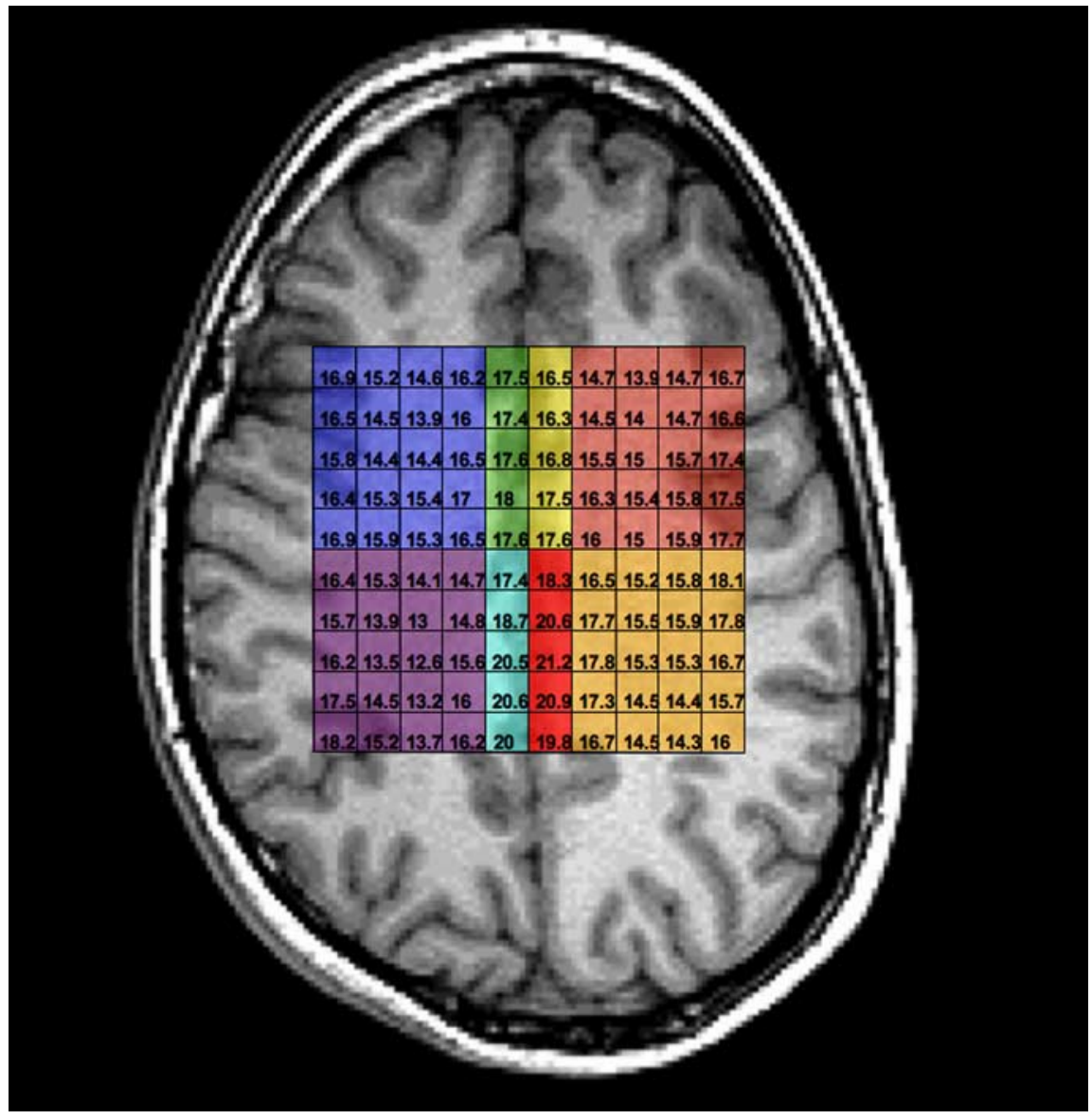

Figure 2. Axial view of MRSI grid showing individual $0.6 \mathrm{~cm} 3$ voxels comprising the eight regions of interest. Image is in radiological convention (left side of image, right hemisphere). Blue, Right anterior white matter; green, right anterior gray matter; yellow, left anterior gray matter; salmon, left anterior white matter; purple, right posterior white matter; light blue, right posterior gray matter; red, left posterior gray matter; tan, left posterior gray matter.

being set at 0.05 . Post hoc bivariate Pearson correlation coefficients were generated to assess relationships between behavioral and spectroscopic variables in the entire sample $(N=56)$, at VIQ $\leq 116(N=25)$, and at VIQ $>116(N=31)$.

\section{Results}

Participants were young adults $(23.9 \pm 4.22)$, well matched by sex (30 males, 26 females) and handedness (males 27 right, 3 left; females 24 right, 2 left). The FSIQ was above average for both sexes $($ males $=118.43 \pm 11.22$; females $=117.73 \pm 8.10)$. Females scored significantly higher than males on the CCI (females 105.09; males 95.28; $\left.t_{(\mathrm{df}=54)}=2.52, p=0.015\right)$. Table 1 shows the demographic statistics as well as significant differences between males and females across intelligence, personality, and spectroscopic measures.

We first assessed the relationships between behavioral and spectroscopic measures across the entire sample. CCI was weakly correlated with FSIQ $(r=0.30, p=0.024)$ in the entire sample. CCI was positively related to openness to experience $(r=0.47$, $p<0.001$ ), as previously reported in the psychometric literature (Sternberg, 2005), but not to other personality domains of the NEO-FFI. Fluency while performing measures of the CCI was weakly related to openness to experience $(r=0.28, p=0.03)$, but fluency while performing the COWAT did not reach significance $[r=0.15$, nonsignificant (ns) $]$. To evaluate the relationships between the eight NAA measures and CCI, we conducted stepwise multiple regression analyses in which the NAA measures were regressed onto CCI. For the total sample, we found that a model including lower right anterior gray matter NAA and higher left anterior gray matter NAA predicted CCI $\left(F_{(2,52)}=6.11, p=0.004, r^{2}=0.20\right)$. We also assessed whether fluency of responses was related to our spectroscopic measures. For the total sample, we found that a model including lower right anterior gray matter NAA predicted total number of responses $\left(F_{(1,52)}=8.38, p=\right.$ $\left.0.006, r^{2}=0.14\right)$. The COWAT was not significantly predicted by any spectroscopic measures. In post hoc analyses, we assessed the bivariate relationships between the COWAT, CCI, fluency measures (across DT tasks), and spectroscopic measures obtained within each of the eight regions of interest. Results are presented in Table 2.

Next, we wished to determine the effects of any intellectual threshold on DT, either as judged by our raters or as measured by fluency of responses. CCI was strongly correlated with FSIQ in the portion of the sample with FSIQ below the threshold of $120(N=31 ; r=0.48, p=$ $0.006)$, but not in the portion of the sample with FSIQ scores $>120(N=25 ; r=0.06$, $p=\mathrm{ns})$, consistent with previous reports (Sternberg, 2005). Follow-up analyses in the total sample revealed that CCI was related to VIQ $(r=0.41, p=0.002)$, but not performance IQ $(r=0.05, \mathrm{~ns})$. Thus, in subsequent analyses we focus on VIQ rather than FSIQ in our analyses of relationships between intelligence, NAA, and CCI. Using a mean split (mean $=116)$ we divided our sample into lower VIQ $(N=25$; mean $=106.16, \mathrm{SD}=8.16)$ and higher VIQ groups $(N=31$; mean $=123.61, \mathrm{SD}=6.10)$ and examined VIQ-CCI correlations in each.

Finally, we wished to determine whether this VIQ threshold mediated the relationship between our measures of DT (CCI, fluency) and biochemistry. In post hoc analyses, for subjects with VIQ of 116 and lower, a model limited to lower right anterior gray matter NAA predicted CCI $\left(F_{(1,22)}=8.71, p=0.008, r^{2}=29\right)$. The COWAT was not predicted by any model of spectroscopic variables at VIQ of 116 and lower. Table 3 shows post hoc bivariate Pearson correlations between behavioral measures and spectroscopic measures for subjects with VIQ $\leq 116$, presented for hypothesis generation. For subjects with VIQ $>116$, a model including lower right anterior gray matter NAA and higher left anterior gray matter NAA predicted CCI $\left(F_{(2,29)}=5.05, p=\right.$ $\left.0.014, r^{2}=0.27\right)$. The COWAT was predicted by a model including lower right posterior gray matter and higher left anterior gray matter for subjects with VIQ $>116\left(F_{(2,29)}=5.18, p=\right.$ $\left.0.013, r^{2}=0.28\right)$. Table 4 shows post hoc bivariate Pearson correlations between behavioral measures and spectroscopic measures for subjects with VIQ $>116$, presented for hypothesis generation. Figure 3 shows the relationship between the CCI and right anterior and left anterior gray matter NAA within cohorts comprised of subjects with VIQ $\leq 116$ and for those with VIQ $>116$. 
Table 1. Demographic variables for the sample

\begin{tabular}{|c|c|c|c|c|c|}
\hline Measure & Mean & SD & $t$ & $p$ & \\
\hline \multirow[t]{2}{*}{ Age } & 23.83 & 4.14 & 0.18 & 0.86 & ns \\
\hline & 24.04 & 4.38 & & & \\
\hline \multirow[t]{2}{*}{ VIQ } & 115.53 & 12.55 & 0.21 & 0.84 & ns \\
\hline & 116.15 & 9.72 & & & \\
\hline \multirow[t]{2}{*}{ PIQ } & 117.70 & 9.65 & 0.89 & 0.38 & ns \\
\hline & 115.58 & 8.26 & & & \\
\hline \multirow[t]{2}{*}{ FSIQ } & 118.43 & 11.22 & 0.27 & 0.79 & ns \\
\hline & 117.73 & 8.10 & & & \\
\hline \multirow[t]{2}{*}{ Openness } & 34.50 & 4.92 & 1.06 & 0.29 & ns \\
\hline & 36.04 & 5.77 & & & \\
\hline \multirow[t]{2}{*}{ COWAT } & 37.23 & 8.27 & 0.76 & 0.45 & ns \\
\hline & 39.75 & 8.12 & & & \\
\hline \multirow[t]{2}{*}{$\mathrm{CCl}$} & 95.228 & 14.59 & -2.52 & 0.015 & * \\
\hline & 105.09 & 14.51 & & & \\
\hline \multirow[t]{2}{*}{ RAWM } & 14.61 & 0.70 & 1.20 & 0.23 & ns \\
\hline & 14.35 & 0.85 & & & \\
\hline \multirow[t]{2}{*}{ RAGM } & 17.64 & 1.86 & 2.32 & 0.024 & * \\
\hline & 16.56 & 1.60 & & & \\
\hline \multirow[t]{2}{*}{ LAGM } & 15.83 & 1.26 & 1.10 & 0.28 & ns \\
\hline & 16.24 & 1.45 & & & \\
\hline \multirow[t]{2}{*}{ LAWM } & 14.01 & 0.86 & 0.87 & 0.39 & ns \\
\hline & 14.21 & 0.83 & & & \\
\hline \multirow[t]{2}{*}{ RPWM } & 14.34 & 0.93 & 0.28 & 0.78 & ns \\
\hline & 14.27 & 0.84 & & & \\
\hline \multirow[t]{2}{*}{ RPGM } & 18.84 & 2.10 & 3.46 & 0.001 & $* * *$ \\
\hline & 17.23 & 1.34 & & & \\
\hline \multirow[t]{2}{*}{ LPGM } & 17.50 & 1.57 & 1.24 & 0.22 & ns \\
\hline & 17.07 & 0.97 & & & \\
\hline \multirow[t]{2}{*}{ LPWM } & 14.07 & 0.78 & 0.53 & 0.60 & ns \\
\hline & 14.18 & 0.78 & & & \\
\hline
\end{tabular}

Upper row, Males; lower row, females. Openness, Openness to experience. ${ }^{*}$ Contrast is significant at the 0.05 level (two-tailed). ${ }^{* * *}$ Contrast is significant at the 0.001 level (two-tailed).

\section{Discussion}

There has been a vigorous discussion regarding the overlap of creativity and intelligence (Sternberg, 2005). We found that NAA in bilateral anterior gray matter predicted CCI in different ways: higher right hemisphere gray matter NAA and lower right hemisphere gray matter NAA each significantly predicted higher levels of creative potential as measured by DT tests. These results suggest that the threshold effect, long documented in the psychometric literature, has neurobiological validity. The interrelationships of creativity, as measured by fluent production of DT, with intellectual, personality, and neurometabolic measures, differ as a function of this threshold. This research is the first to show that neurometabolite levels predict DT, and that different biochemical organization supports creative potential in lower versus higher verbal intelligence cohorts.

We have used a measure of DT to assess creative cognition in a cohort of normal college aged students. This should not be interpreted to equate DT with creativity. Rather, it is one of many possible cognitive measures, in this case designed to tap deliberate processing modes of the brain within which cognitive knowledge domains are accessed, characteristic of scientific or entrepreneurial creativity (Dietrich, 2004). What the current research demonstrates is that mere ideational combination is not predicted by biochemical substrates localizable to the anterior medial gray matter. Indeed, verbal fluency, as measured by the COWAT, required subjects to produce ideational combinations at a rapid pace and was largely unrelated to either DT or spectroscopic measures. However, when subjects were required to both produce and select ideational combinations, both the fluency of responses and judges' ratings were predicted by medial gray matter NAA. Thus, the DT measure, as used currently, appears to conform to the notion that "a selection process is required to determine which ideas are truly creative" (Dietrich, 2004).

The gray matter values come from the cingulate gyrus. The posterior aspects of the anterior cingulate cortex has been associated with monitoring unfavorable performance outcomes, response errors, and response conflicts (Ridderinkhof et al., 2004). One theory postulates that the dorsal anterior cingulate codes outcome and error-related information by triggering phasic changes in the activity of the midbrain dopamine system to improve task performance according to reinforcement learning (Holroyd et al., 2004). An alternate theory postulates a role for the dorsal anterior cingulate in monitoring response conflict by activation of additional brain regions designed to increase cognitive control (Botvinick et al., 2001). Of note, the highest convergence of activity for which the cingulate was noted to be involved in monitoring processes tended to cluster in transition zones between cingulate, association (Brodmann area 8), and premotor territories (area 6), regions within our anterior spectroscopic voxels.

Exactly how does variation in NAA concentration affect brain activity in normals? A rapidly growing literature links NAA to intelligence, working memory, attention, and memory both in health and disease (Ross and Sachdev, 2004), but the mechanisms underlying these relationships remain elusive. In the adult brain, there is substantial evidence that NAA is a marker of mitochondrial functioning, is involved in myelin lipid turnover, participates in axon-glial signaling, and may be involved in brain nitrogen balance (Moffett et al., 2007). Substantial basic research has yet to unravel the complex role that NAA plays in higher cognitive functioning, including creativity, beyond mere correlation.

There are two major theories regarding hemispheric interactions as related to creative performance. The first theory postulates higher interconnectivity in the right hemisphere than the left with activation of large semantic networks facilitated by increased white matter connectivity (Jung-Beeman, 2005). These large overlapping fields allow weak signals to summate, potentially facilitating unique combinations. In our sample, lower IQ subjects had stronger inverse CCI-NAA relationships, perhaps reflecting central (i.e., cingulate) disinhibition of large right hemisphere semantic networks. In contrast, in the higher VIQ cohort, weak positive CCI-NAA relationships were seen, perhaps reflecting central (i.e., cingulate) facilitation of more refined access to discrete left hemisphere semantic networks. A second group theorizes that neural efficiency plays a significant role in guiding the cortical allocation of resources toward creative idea generation by constraining top down control of information processing (Fink and Neubauer, 2006). In our cohort, it is possible that the higher VIQ subjects are more efficiently allocating neural resources, reflected in the CCI-NAA relationship, whereas lower VIQ subjects have less efficient top down control. What these studies, and the current one, highlight is the inescapable fact that a construct as complex as creativity will never be localized in the brain, be it the right hemisphere, anterior cingulate cortex, or other locus. Rather, individual findings will be dependent on the task used as a "proxy" measure, the population under scrutiny, and even methodological issues related to structural versus functional characteristics of the brain.

The strengths of the current study include (1) the inclusion of a young, healthy cohort, (2) use of well established psychometric measures of DT, verbal fluency, intelligence, and personality, and 
Table 2. Post hoc bivariate Pearson correlations between behavioral measures and spectroscopic measures from eight regions of interest for the entire sample $(N=56)$

\begin{tabular}{|c|c|c|c|c|c|c|c|}
\hline & COWAT & UOT creativity & Free draw creativity & 4 line creativity & UOT fluency & Free draw fluency & 4 line fluency \\
\hline COWAT & 1 & & & & & & \\
\hline \multirow[t]{2}{*}{ UOT creativity } & 0.27 & 1 & & & & & \\
\hline & 0.05 & & & & & & \\
\hline \multirow[t]{2}{*}{ Free draw creativity } & 0.11 & 0.35 & 1 & & & & \\
\hline & ns & 0.007 & & & & & \\
\hline \multirow[t]{2}{*}{4 line creativity } & 0.22 & 0.46 & 0.71 & 1 & & & \\
\hline & ns & 0.001 & 0.001 & & & & \\
\hline \multirow[t]{2}{*}{ UOT fluency } & -0.06 & 0.52 & 0.10 & 0.18 & 1 & & \\
\hline & ns & 0.001 & ns & ns & & & \\
\hline \multirow[t]{2}{*}{ Free draw fluency } & -0.13 & 0.12 & 0.40 & 0.26 & 0.07 & 1 & \\
\hline & ns & ns & 0.002 & 0.05 & ns & & \\
\hline \multirow[t]{2}{*}{4 line fluency } & 0.25 & 0.23 & 0.54 & 0.73 & 0.14 & 0.35 & 1 \\
\hline & ns & ns & 0.001 & 0.001 & ns & ns & \\
\hline \multirow[t]{2}{*}{ RAWM NAA } & -0.21 & 0.15 & -0.10 & -0.03 & 0.10 & 0.14 & 0.02 \\
\hline & ns & ns & ns & ns & ns & ns & ns \\
\hline \multirow[t]{2}{*}{ RPWM NAA } & -0.05 & 0.10 & -0.07 & 0.01 & 0.03 & 0.01 & 0.02 \\
\hline & ns & ns & ns & ns & ns & ns & ns \\
\hline \multirow[t]{2}{*}{ LAWM NAA } & 0.01 & 0.29 & 0.23 & 0.25 & 0.08 & 0.13 & 0.09 \\
\hline & ns & 0.03 & ns & ns & ns & ns & ns \\
\hline \multirow[t]{2}{*}{ LPWM NAA } & -0.13 & 0.17 & 0.11 & 0.12 & 0.17 & -0.12 & 0.03 \\
\hline & ns & ns & ns & ns & ns & ns & ns \\
\hline \multirow[t]{2}{*}{ RAGM NAA } & -0.16 & -0.26 & -0.21 & -0.32 & -0.23 & -0.12 & -0.31 \\
\hline & ns & 0.05 & ns & 0.02 & ns & ns & 0.02 \\
\hline \multirow[t]{2}{*}{ RPGM NAA } & -0.22 & -0.31 & -0.19 & -0.19 & -0.09 & -0.15 & -0.27 \\
\hline & ns & 0.02 & ns & ns & ns & ns & 0.04 \\
\hline \multirow[t]{2}{*}{ LAGM NAA } & 0.04 & 0.16 & 0.23 & 0.01 & -0.09 & -0.04 & 0.12 \\
\hline & ns & ns & ns & ns & ns & ns & ns \\
\hline \multirow[t]{2}{*}{ LPGM NAA } & -0.16 & -0.09 & 0.08 & -0.07 & 0.04 & 0.05 & -0.11 \\
\hline & ns & ns & ns & ns & ns & ns & ns \\
\hline
\end{tabular}

UOT creativity, UOT-judged creativity ratings; Free draw creativity, free drawing task-judged creativity ratings; 4 line creativity, four line drawing task-judged creativity ratings; U0T fluency, U0T-number of responses; Free draw creativity, Free drawing task-number of responses; 4 line creativity, Four line drawing task-number of responses. Values in bold and italic indicate $p<0.05$.

Table 3. Post hoc bivariate Pearson correlations between behavioral measures and spectroscopic measures from eight regions of interest for subjects with VIQ $\leq 116$

\begin{tabular}{|c|c|c|c|c|c|c|c|}
\hline & COWAT & UOT creativity & Free draw creativity & 4 line creativity & UOT fluency & Free draw fluency & 4 line fluency \\
\hline COWAT & 1 & & & & & & \\
\hline \multirow[t]{2}{*}{ UOT creativity } & 0.17 & 1 & & & & & \\
\hline & ns & & & & & & \\
\hline \multirow[t]{2}{*}{ Free draw creativity } & 0.01 & -0.01 & 1 & & & & \\
\hline & ns & ns & & & & & \\
\hline \multirow[t]{2}{*}{4 line creativity } & 0.25 & 0.15 & 0.72 & 1 & & & \\
\hline & ns & ns & 0.001 & & & & \\
\hline \multirow[t]{2}{*}{ UOT fluency } & -0.02 & 0.54 & -0.06 & 0.06 & 1 & & \\
\hline & ns & 0.005 & ns & ns & & & \\
\hline \multirow[t]{2}{*}{ Free draw fluency } & -0.05 & -0.06 & 0.42 & 0.23 & -0.02 & 1 & \\
\hline & ns & ns & 0.04 & ns & ns & & \\
\hline \multirow[t]{2}{*}{4 line fluency } & 0.36 & 0.10 & 0.70 & 0.82 & 0.01 & 0.37 & 1 \\
\hline & ns & ns & 0.001 & 0.001 & ns & ns & \\
\hline \multirow[t]{2}{*}{ RAWM NAA } & -0.10 & 0.28 & -0.21 & -0.12 & 0.11 & -0.04 & -0.17 \\
\hline & ns & ns & ns & ns & ns & ns & ns \\
\hline \multirow[t]{2}{*}{ RPWM NAA } & 0.12 & 0.22 & -0.12 & -0.02 & -0.11 & -0.20 & -0.09 \\
\hline & ns & ns & ns & ns & ns & ns & ns \\
\hline \multirow[t]{2}{*}{ LAWM NAA } & -0.31 & 0.22 & 0.20 & 0.18 & 0.07 & 0.17 & 0.07 \\
\hline & ns & ns & ns & ns & ns & ns & ns \\
\hline \multirow[t]{2}{*}{ LPWM NAA } & -0.22 & 0.10 & 0.03 & 0.08 & 0.22 & -0.12 & 0.04 \\
\hline & ns & ns & ns & ns & ns & ns & ns \\
\hline \multirow[t]{2}{*}{ RAGM NAA } & 0.08 & -0.20 & -0.46 & -0.50 & -0.08 & -0.17 & -0.52 \\
\hline & ns & ns & 0.02 & 0.01 & ns & ns & 0.008 \\
\hline \multirow[t]{2}{*}{ RPGM NAA } & 0.12 & -0.34 & -0.39 & -0.32 & 0.07 & -0.32 & -0.42 \\
\hline & ns & ns & ns & ns & ns & ns & 0.04 \\
\hline \multirow[t]{2}{*}{ LAGM NAA } & -0.26 & 0.04 & 0.13 & -0.21 & -0.08 & -0.16 & -0.03 \\
\hline & ns & ns & ns & ns & ns & ns & ns \\
\hline \multirow[t]{2}{*}{ LPGM NAA } & -0.25 & -0.17 & -0.12 & -0.40 & 0.17 & -0.23 & -0.20 \\
\hline & ns & ns & ns & 0.05 & ns & ns & ns \\
\hline
\end{tabular}

UOT creativity, UOT-judged creativity ratings; Free draw creativity, free drawing task-judged creativity ratings; 4 line creativity, four line drawing task-judged creativity ratings; U0T fluency, U0T-number of responses; Free draw creativity, free drawing task-number of responses; 4 line creativity, four line drawing task-number of responses. Values in bold and italic indicate $p<0.05$. 
Table 4. Post hoc bivariate Pearson correlations between behavioral measures and spectroscopic measures from eight regions of interest for subjects with VIQ $>116$

\begin{tabular}{|c|c|c|c|c|c|c|c|}
\hline & COWAT & UOT creativity & Free draw creativity & 4 line creativity & UOT fluency & Free draw fluency & 4 line fluency \\
\hline COWAT & 1 & & & & & & \\
\hline \multirow[t]{2}{*}{ UOT creativity } & 0.23 & 1 & & & & & \\
\hline & ns & & & & & & \\
\hline \multirow[t]{2}{*}{ Free draw creativity } & 0.05 & 0.57 & 1 & & & & \\
\hline & ns & 0.001 & & & & & \\
\hline \multirow[t]{2}{*}{4 line creativity } & 0.12 & 0.69 & 0.68 & 1 & & & \\
\hline & ns & 0.001 & 0.001 & & & & \\
\hline \multirow[t]{2}{*}{ UOT fluency } & -0.13 & 0.51 & 0.26 & 0.31 & 1 & & \\
\hline & ns & 0.004 & ns & ns & & & \\
\hline \multirow[t]{2}{*}{ Free draw fluency } & -0.29 & 0.16 & 0.33 & 0.23 & 0.14 & 1 & \\
\hline & ns & ns & ns & ns & ns & & \\
\hline \multirow[t]{2}{*}{4 line fluency } & 0.14 & 0.37 & 0.39 & 0.63 & 0.32 & 0.34 & 1 \\
\hline & ns & 0.04 & 0.03 & 0.001 & ns & ns & \\
\hline \multirow[t]{2}{*}{ RAWM NAA } & -0.21 & 0.16 & 0.06 & 0.11 & 0.11 & 0.33 & 0.25 \\
\hline & ns & ns & ns & ns & ns & ns & ns \\
\hline \multirow[t]{2}{*}{ RPWM NAA } & -0.23 & -0.07 & -0.05 & 0.03 & 0.09 & 0.18 & 0.19 \\
\hline & ns & ns & ns & ns & ns & ns & ns \\
\hline \multirow[t]{2}{*}{ LAWM NAA } & 0.19 & 0.33 & 0.23 & 0.30 & 0.07 & 0.08 & 0.11 \\
\hline & ns & ns & ns & ns & ns & ns & ns \\
\hline \multirow[t]{2}{*}{ LPWM NAA } & -0.06 & 0.27 & 0.19 & 0.18 & 0.10 & -0.14 & 0.01 \\
\hline & ns & ns & ns & ns & ns & ns & ns \\
\hline \multirow[t]{2}{*}{ RAGM NAA } & -0.16 & -0.20 & 0.03 & -0.16 & -0.35 & -0.01 & -0.14 \\
\hline & ns & ns & ns & ns & ns & ns & ns \\
\hline \multirow[t]{2}{*}{ RPGM NAA } & -0.37 & -0.24 & 0.01 & -0.05 & -0.25 & 0.01 & -0.11 \\
\hline & 0.04 & ns & ns & ns & ns & ns & ns \\
\hline \multirow[t]{2}{*}{ LAGM NAA } & 0.32 & 0.38 & 0.40 & 0.25 & -0.07 & 0.11 & 0.31 \\
\hline & ns & 0.04 & 0.03 & ns & ns & ns & ns \\
\hline \multirow[t]{2}{*}{ LPGM NAA } & -0.05 & 0.06 & 0.36 & 0.34 & -0.15 & 0.35 & 0.05 \\
\hline & ns & ns & 0.04 & ns & ns & ns & ns \\
\hline
\end{tabular}

UOT creativity, UOT-judged creativity ratings; Free draw creativity, free drawing task-judged creativity ratings; 4 line creativity, four line drawing task-judged creativity ratings; U0T fluency, U0T-number of responses; Free draw creativity, free drawing task-number of responses; 4 line creativity, four line drawing task-number of responses. Values in bold and italic indicate $p<0.05$.

(3) excellent matching on major demographic and psychometric factors of interest including sex, handedness, and IQ. Major weaknesses include a lack of fine-grained spectroscopic resolution given use of SI protocol, inadequate distribution of intellectual capacity below the average range of functioning (i.e., FSIQ <90), and insufficient power to assess other hypotheses relevant to NAAcognition relationships. The first weakness is particularly glaring given the desire to "localize" creative behavior within the cingulate gyrus (i.e., Brodmann area 32 vs 24). Similarly, our limited sampling of the full range of intellectual ability is a common hazard when sampling from college cohorts. However, as the relationships between intellectual and creative capacity have been noted to disentangle at IQ's $>120$, focus on this end of the distribution appears warranted given the current study aims. Finally, reliance on measures of DT as "proxy" measures of creativity is an ongoing weakness both in this study and in the field. We use this measure cautiously, and with the knowledge that creativity is a complex construct comprised of cognitive factors including (but not limited to) DT, convergent thinking, insight, etc. Once we address these basic cognitive
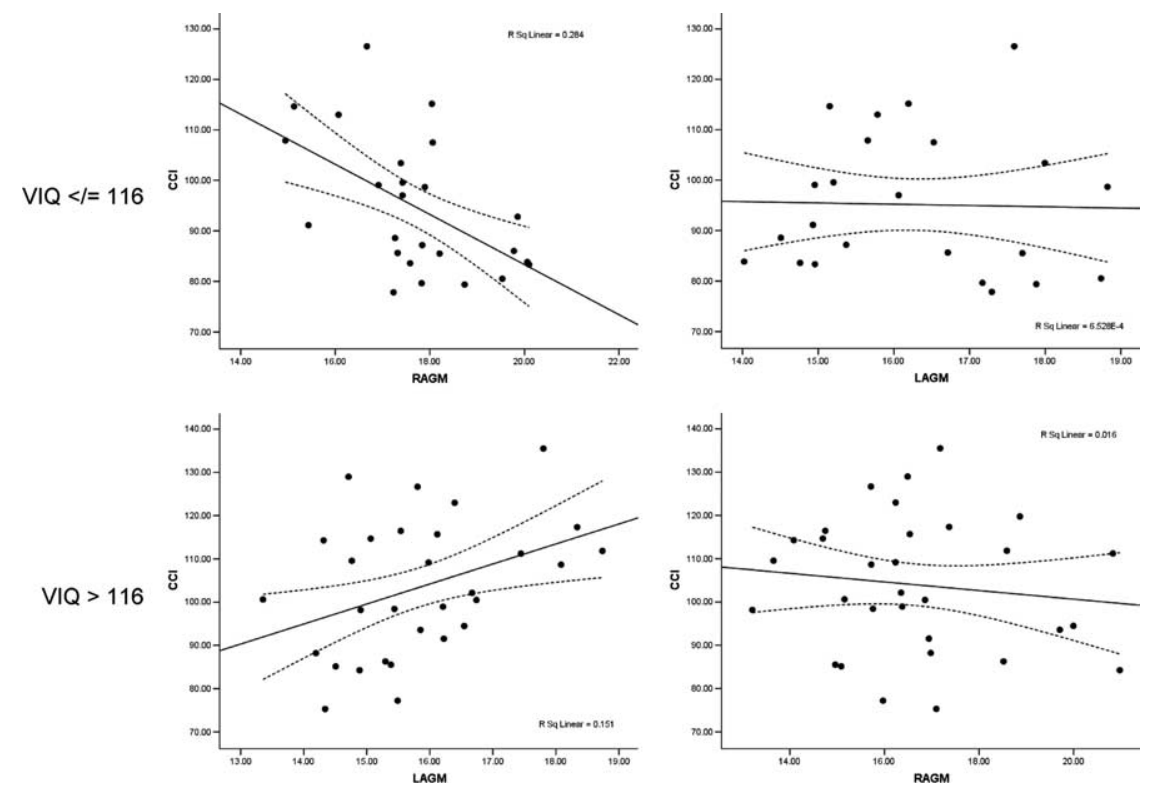

Figure 3. Scatter plot of relationships between NAA and CCl in lower and higher VIQ subjects. Top left, Significant relationship $\left(r_{2}=0.28\right)$ between $\mathrm{CCl}$ and right anterior gray matter in $\mathrm{VIQ}</=116$; top right, nonsignificant relationship between $\mathrm{CCl}$ and left anterior gray matter in VIQ $</=116$; lower left, significant relationship $\left(r_{2}=0.15\right)$ between CCl and left anterior gray matter in VIQ >116; lower right, nonsignificant relationship between $\mathrm{CCl}$ and right anterior gray matter in VIQ $>116$. processes, and link them meaningfully to creative cognition, we might hope to make significant progress in better understanding the interplay of novelty and usefulness that bounds the neuroscience of creativity. 


\section{References}

Amabile TM (1982) Social psychology of creativity: a consensual assessment technique. J Pers Soc Psychol 43:997-1013.

Asari T, Konishi S, Jimura K, Chikazoe J, Nakamura N, Miyashita Y (2008) Right temporopolar activation associated with unique perception. Neuroimage 41:145-152.

Bechtereva NP, Korotkov AD, Pakhomov SV, Roudas MS, Starchenko MG, Medvedev SV (2004) PET study of brain maintenance of verbal creative activity. Int J Psychophysiol 53:11-20.

Botvinick MM, Braver TS, Barch DM, Carter CS, Cohen JD (2001) Conflict monitoring and cognitive control. Psych Rev 108:624-652.

Carlsson I, Wendt PE, Risberg J (2000) On the neurobiology of creativity. Differences in frontal activity between high and low creative subjects. Neuropsychologia 38:873-885.

Dietrich A (2003) Functional neuroanatomy of altered states of consciousness: the transient hypofrontality hypothesis. Conscious Cogn $12: 231-256$

Dietrich A (2004) The cognitive neuroscience of creativity. Psychon Bull Rev 11:1011-1026.

Dietrich A (2007) Who's afraid of a cognitive neuroscience of creativity? Methods 42:22-27.

Fink A, Neubauer AC (2006) EEG alpha oscillations during the performance of verbal creativity tasks: differential effects of sex and verbal intelligence. Int J Psychophysiol 62:46-53.

Gray JR, Thompson PM (2004) Neurobiology of intelligence: science and ethics. Nat Rev Neurosci 5:471-482.

Holroyd CB, Nieuwenhuis S, Yeung N, Nystrom L, Mars RB, Coles MG,
Cohen JD (2004) Dorsal anterior cingulate cortex shows fMRI response to internal and external error signals. Nat Neurosci 7:497-498.

Howard-Jones PA, Blakemore SJ, Samuel EA, Summers IR, Claxton G (2005) Semantic divergence and creative story generation: an fMRI investigation. Brain Res Cogn Brain Res 25:240-250.

Jausovec N (2000) Differences in cognitive processes between gifted, intelligent, creative, and average individuals while solving comple problems: an EEG study. Intelligence 28:213-237.

Jausovec N, Jausovec K (2000) Differences in resting EEG related to ability. Brain Topogr 12:229-240.

Jung-Beeman M (2005) Bilateral brain processes for comprehending natural language. Trends Cogn Sci 9:512-518.

Jung RE, Haier RJ (2007) The parieto-frontal integration theory (P-FIT) of intelligence: converging neuroimaging evidence. Behav Brain Sci 30:135-154.

Lezak MD, Howieson DB, Loring DW, Hannay HJ, Fischer JS (2004) Neuropsychological assessment. New York: Oxford UP.

Moffett JR, Ross B, Arun P, Madhavarao CN, Namboodiri AM (2007) $\mathrm{N}$-Acetylaspartate in the CNS: from neurodiagnostics to neurobiology. Prog Neurobiol 81:89-131.

Ridderinkhof KR, Ullsperger M, Crone EA, Nieuwenhuis S (2004) The role of the medial frontal cortex in cognitive control. Science 306:443-447.

Ross AJ, Sachdev PS (2004) Magnetic resonance spectroscopy in cognitive research. Brain Res Brain Res Rev 44:83-102.

Simonton DK (2003) Scientific creativity as constrained stochastic behavior: the integration of product, person, and process perspectives. Psychol Bull 129:475-494.

Sternberg RJ (2005) Handbook of creativity. New York: Cambridge UP. 